\title{
Weak Convergence of Ishikawa Iteration with Error for Pseudo Contractive Mappings in Hilbert Spaces
}

\author{
Dingping Wu \\ College of Mathematics, Chengdu University of Information Technology \\ Chengdu 610225, China \\ E-mail:wdp68@163.com
}

Received: September 9, 2011 Accepted: September 23, 2011 Published: November 1, 2011

doi:10.5539/jmr.v3n4p44 URL: http://dx.doi.org/10.5539/jmr.v3n4p44

This work is supported by Chengdu University of Information Technology Introduced Fund Professionals (No.KYTZ201004).

\begin{abstract}
Our purpose in this paper is to consider a sequence $\left\{x_{n}\right\}$ defined as (2) and a asymptotically $k_{n}$ - strict pseudocontractive mapping in the intermediate sense Ishikawa iterative process with errors. The results presented in this paper mainly improved and extend the corresponding results announced in [D.R.Sahu, 2009; T.H.Kim, 2008; K.Nammanee, 2000; T.H.Kim, 2008].
\end{abstract}

Keywords: Demiclosedness principle, Asymptotically strict pseudocontrative mapping, Fixed point, Ishikawa iterative process

\section{Introduction}

The class of asymptotically nonexpansive mappings was introduced as an important generalization of the class of nonexpansive mappingsfand the existence of fixed points of asymptotically nonexpansive mappings was proved by Goebel and Kirk [1972] as below:

Theorem 1. If $C$ is a nonempty closed convex bounded subset of a uniformly convex Banach Space, then every asymptotically nonexpansive mapping $T: C \longrightarrow C$ has a fixed point in $C$.

Mann iteration process:

$$
x_{n+1}=\left(1-\alpha_{n}\right) x_{n}+\alpha_{n} T^{n} x_{n}, \quad n \in \mathbb{N} .,
$$

for (1) the approximation of fixed points of asymptotically nonexpansive mappings was developed and it's weak convergence was obtained by Schu [1991].

Iterative methods for approximation of fixed points of asymptotically nonexpansive mappings have been widely studied by authors [C.E.Chidume, 2003; S.S.Chang, 2001; S.H.Khan, 2005; Z.Q.Liu, 2004; K.Nammanee, 2000; B.E.Rhoades, 1994; M.O.Osilike, 2000; J.Schu, 1991; L.Wang, 2006].

The class of asymptotically nonexpansive mappings in the intermediate sense was introduced by Bruck, Kuczumow and Reich [1993] .

In 2008, Kim and Xu [2008] introduced the concept of asymptotically $k$-strict pseudocontractive mappings in Hilbert space.

In 2009, Sahu and Xu [2009] study some properties and convergence of (1) for the class of asymptotically $k$-strict pseudocontractive mappings in the intermedidate sense which are not necessarily Lipschitzian.

Our purpose in this paper is to consider a sequence $\left\{x_{n}\right\}$ defined as (2) and a asymptotically $k_{n}$ - strict pseudocontractive mapping in the intermediate sense Ishikawa iterative process with errors. The results presented in this paper mainly improved and extend the corresponding results announced in [D.R.Sahu, 2009; T.H.Kim, 2008; K.Nammanee, 2000; T.H.Kim, 2008].

$$
\left\{\begin{array}{c}
x_{n+1}=\left(1-\alpha_{n}\right) x_{n}+\alpha_{n} T^{n} y_{n}+\sigma_{n} \\
y_{n}=\left(1-\beta_{n}\right) x_{n}+\beta_{n} T^{n} x_{n}+\tau_{n}
\end{array}\right.
$$




\section{Preliminaries}

Let $C$ be a nonempty subset of a normed space $X$ and $T: C \rightarrow C$ a mapping. We need the following concepts,

(i) $T$ is nonexpansive if,

$$
\|T x-T y\| \leq\|x-y\|
$$

for all $x, y \in C$.

(ii) $T$ is asymptotically nonexpansive(cf.[K.Goebel, 1972])if there exists a sequence $k_{n}$ of positive numbers satisfying the property

$$
\lim _{n \rightarrow \infty} k_{n}=1 \text { and }\left\|T^{n} x-T^{n} y\right\| \leq k_{n}\|x-y\|
$$

for all integers $n \geq 1$ and $x, y \in C$.

(iii) $T$ is uniformly Lipschitzian if there exists a constant $L>0$ such that,

$$
\left\|T^{n} x-T^{n} y\right\| \leq L\|x-y\|
$$

for all integers $n \geq 1$ and $x, y \in C$.

(iv) $T$ is asymptotically nonexpansive in the intermediate sense [R.E.Bruck, 1993] provided $T$ is uniformly continuous and,

$$
\limsup _{n \rightarrow \infty} \sup _{x, y \in C}\left(\left\|T^{n} x-T^{n} y\right\|-\|x-y\|\right) \leq 0 .
$$

It is clear that every nonexpansive mapping is asymptotically nonexpansive and every asymptotically nonexpansive mapping is uniformly Lipschitzian.

Let $H$ be a real Hilbert space with inner product $\langle\cdot, \cdot\rangle$ and norm $\|\cdot\|$, respectively and let $C$ be a closed convex subset of $H, \omega_{\omega}\left(\left\{x_{n}\right\}\right)$ denotes the weak $\omega$ - limit set of $\left\{x_{n}\right\}$.

Definition 1 Let $C$ be a nonempty subset of a Hilbert space $H$. A mapping $T: C \longrightarrow C$ is said to be an asymptotically $k_{n}$-strict pseudocontractive mapping in the intermediate sense with sequence $\left\{\gamma_{n}\right\}$ if there exist sequences $\left\{k_{n}\right\}$ in $[0,1)$ and $\left\{\gamma_{n}\right\},\left\{c_{n}\right\}$ in $[0, \infty)$ with $\limsup _{n \rightarrow \infty} k_{n}=k<1, \lim _{n \rightarrow \infty} \gamma_{n}=\lim _{n \rightarrow \infty} c_{n}=0$ such that

$$
\left\|T^{n} x-T^{n} y\right\|^{2} \leq\left(1+\gamma_{n}\right)\|x-y\|^{2}+k_{n}\left\|x-T^{n} x-\left(y-T^{n} y\right)\right\|^{2}+c_{n},
$$

for all $x, y \in C$ and $n \in \mathbb{N}$.

we need some facts and tools which are listed as follows,

Lemma 1. ([M.O.Osilike, 2000; K.K.Tan, 1992]) Let $\left\{\gamma_{n}\right\},\left\{\beta_{n}\right\},\left\{\delta_{n}\right\}$ be three sequences of nonnegative numbers satisfying the recursive inequality,

$$
\delta_{n+1} \leq \delta_{n} \beta_{n}+\gamma_{n}, \forall n \in \mathbb{N}
$$

If $\beta_{n} \geq 1, \Sigma_{n=1}^{\infty}\left(\beta_{n}-1\right)<\infty$ and $\Sigma_{n=1}^{\infty} \gamma_{n}<\infty$ then $\lim _{n \rightarrow \infty} \delta_{n}$ exists.

Lemma 2. (Agarwal, O'Regon. [2007]) Let $\left\{x_{n}\right\}$ be a bounded sequence in a reflexive Banach space X. if $\omega_{\omega}\left(\left\{x_{n}\right\}\right)=\{x\}$, then $x_{n}$ converges weakly to $x$.

Lemma 3. Let $H$ be a real Hilbert space. Then the following hold:

(a) $\|x-y\|^{2}=\|x\|^{2}-\|y\|^{2}-2\langle x-y, y\rangle, \forall x, y \in H$;

(b) $\|(1-t) x+t y\|^{2}=(1-t)\|x\|^{2}+t\|y\|^{2}-t(1-t)\|x-y\|^{2}, \forall x, y \in H$;

(c) let $\left\{x_{n}\right\} \in H$ such that $x_{n}$ weak converges to $x$, then $\lim _{\sup _{n \rightarrow \infty}}\left\|x_{n}-y\right\|^{2}=\lim \sup _{n \rightarrow \infty}\left\|x_{n}-x\right\|^{2}+\|x-y\|, \forall y \in H$.

Lemma 4. ([D.R.Sahu, 2009]) Let $C$ be a nonempty subset of a Hilbert space $H, T: C \rightarrow C$ an asymptotically $k-$ strict pseudocontractive mapping in the intermediate sense with sequence $\left\{\gamma_{n}\right\}$. Then

$$
\left\|T^{n} x-T^{n} y\right\| \leq \frac{1}{1-k}\left(k\|x-y\|+\sqrt{\left.\left(1+(1-k) \gamma_{n}\right)\|x-y\|^{2}+(1-k) c_{n}\right)}\right.
$$

for all $x, y \in C$ and $n \in \mathbb{N}$. 
Lemma 5. ([D.R.Sahu, 2009]) Let $C$ be a nonempty subset of a Hilbert space $H, T: C \rightarrow C$ a uniformly continuous asymptotically $k$ - strict pseudocontractive mapping in the intermediate sense with sequence $\left\{\gamma_{n}\right\}$, let $\left\{x_{n}\right\}$ be a bounded sequence in $C$ such that $\left\|x_{n+1}-x_{n}\right\| \rightarrow 0$ and $\left\|x_{n}-T^{n} x_{n+1}\right\| \rightarrow 0,(n \rightarrow \infty)$ Then $\left\|x_{n}-T x_{n}\right\| \rightarrow 0$,as $(n \rightarrow \infty)$.

\section{Main results}

Theorem 2. Let $C$ be be a nonempty closed convex subset of a Hilbert space $H, T: C \rightarrow C$ a continuous asymptotically $k_{n}$ - strict pseudo -contractive mapping in the intermediate sense with sequence $\left\{\gamma_{n}\right\}$. Then $F(T)$ is closed and convex and $I-T$ is demiclosed at zero.

Proof. It is easy to see that $F(T)$ is closed and convex.

We prove that $I-T$ is demiclosed at zero. Assume that $\left\{x_{n}\right\}$ be a sequence in $C$. By Lemma 4 . we obtain

$$
\left\|T^{m} x_{n}-T^{m} x\right\|<M
$$

for all $n, m \in N$ and some constant $M>0$.

Let $f(x)=\lim \sup _{n \rightarrow \infty}\left\|x_{n}-x\right\|^{2}$, for all $x \in H$. Since $\left\{x_{n}\right\}$ weakly converges to $x$,

$$
f(y)=f(x)+\|x-y\|^{2} \quad \text { for all } y \in H .
$$

Hence we have

$$
\begin{aligned}
& f\left(T^{m} x\right)=\limsup _{n \rightarrow \infty}\left\|x_{n}-T^{m} x\right\|^{2} \\
& \leq \limsup _{n \rightarrow \infty}\left(\left(1+\gamma_{m}\right)\left\|x_{n}-x\right\|^{2}+k_{m}\left\|x_{n}-T^{m} x_{n}-\left(x-T^{m} x\right)\right\|^{2}+c_{m}\right)+ \\
& \limsup _{n \rightarrow \infty}\left(\left\|x_{n}-T^{m} x_{n}\right\|^{2}+2\left\|x_{n}-T^{m} x_{n}\right\| M\right) \\
& \leq f(x)+k \limsup _{n \rightarrow \infty}\left\|x_{n}-T^{m} x_{n}-\left(x-T^{m} x\right)\right\|^{2}+\limsup _{n \rightarrow \infty}\left(\left\|x_{n}-T^{m} x_{n}\right\|^{2}\right. \\
& \left.+2\left\|x_{n}-T^{m} x_{n}\right\| M\right)+f(x) \gamma_{m}+c_{m}
\end{aligned}
$$

for each $m \in N$. Since $f(x)+\left\|x-T^{m} x\right\|^{2}=f\left(T^{m} x\right)$,

$$
\left\|x-T^{m} x\right\|^{2} \leq f(x) \gamma_{m}+c_{m}+k \limsup _{n \rightarrow \infty}\left\|x_{n}-T^{m} x_{n}-\left(x-T^{m} x\right)\right\|^{2}+\limsup _{n \rightarrow \infty}\left(\left\|x_{n}-T^{m} x_{n}\right\|^{2}+2\left\|x_{n}-T^{m} x_{n}\right\| M\right) .
$$

By $\lim \sup _{n \rightarrow \infty}\left\|x_{n}-T^{m} x_{n}\right\|=0$, we have

$$
\limsup _{m \rightarrow \infty}\left\|x-T^{m} x\right\|^{2} \leq k \limsup _{m \rightarrow \infty}\left\|x-T^{m} x\right\|^{2} .
$$

It follows that $T^{m} x \rightarrow x(m \rightarrow \infty)$ and $(I-T) x=0$.

Theorem 3. Let $C$ be a nonempty subset of a Hilbert space $H$ and $T: C \rightarrow C$ be uniformly continuous asymptotically $k_{n}-$ pseudocontracitive in the intermediate sense with sequence $\gamma_{n}$ such that $F(T) \neq \phi$. Assume $\left\{\sigma_{n}\right\},\left\{\tau_{n}\right\} \subset C,\left\{\alpha_{n}\right\},\left\{\beta_{n}\right\} \subset$ $(0,1)$ such that $0<\delta \leq \alpha_{n}, \beta_{n} \leq 1-k_{n}-\delta<1$ and $\left\{x_{n}\right\}$ defined as (2) satisfy the following conditions:

(i) $\sum_{n=1}^{\infty} \gamma_{n}<\infty, \sum_{n=1}^{\infty} c_{n}<\infty, \sum_{n=1}^{\infty} k_{n}<\infty, \sum_{n=1}^{\infty}\left\|\sigma_{n}\right\|^{2}<\infty$ and $\sum_{n=1}^{\infty}\left\|\tau_{n}\right\|^{2}<\infty$;

(ii) $\left\{x_{n}\right\}$ be bounded.

Then $\left\{x_{n}\right\}$ converges weakly to an element of $F(T)$.

Proof. First we show that $\left\{\left\|y_{n}-T^{n} y_{n}\right\|\right\}$ be bounded.

$$
\begin{aligned}
\left\|y_{n}-p\right\|^{2}= & \left\|\left(1-\beta_{n}\right)\left(x_{n}+\tau_{n}-p\right)+\beta_{n}\left(T^{n} x_{n}+\tau_{n}-p\right)\right\|^{2} \\
= & \left(1-\beta_{n}\right)\left\|x_{n}+\tau_{n}-p\right\|^{2}+\beta_{n}\left\|T^{n} x_{n}+\tau_{n}-p\right\|^{2}-\beta_{n}\left(1-\beta_{n}\right)\left\|T^{n} x_{n}-x_{n}\right\|^{2} \\
\leq & \left(1-\beta_{n}\right)\left[\left\|x_{n}-p\right\|^{2}+2\left\langle\tau_{n}, x_{n}-p\right\rangle+\left\|\tau_{n}\right\|^{2}\right]+\beta_{n}\left[\left\|T^{n} x_{n}-p\right\|^{2}\right. \\
& \left.+2\left\langle\tau_{n}, T^{n} x_{n}-p\right\rangle+\left\|\tau_{n}\right\|^{2}\right]-\beta_{n}\left(1-\beta_{n}\right)\left\|T^{n} x_{n}-x_{n}\right\|^{2} \\
= & \left(1-\beta_{n}\right)\left\|x_{n}-p\right\|^{2}+\beta_{n}\left\|T^{n} x_{n}-p\right\|^{2}+\left\|\tau_{n}\right\|^{2}-\beta_{n}\left(1-\beta_{n}\right)\left\|T^{n} x_{n}-x_{n}\right\|^{2}+\sigma .
\end{aligned}
$$


where $\sigma:=2\left\langle\left(1-\beta_{n}\right)\left(x_{n}-p\right)+\beta_{n}\left(T^{n} x_{n}-p\right), \tau_{n}\right\rangle$, we simply $\sigma$ as follows.

$$
\begin{aligned}
\sigma & =2\left\langle\left(1-\beta_{n}\right)\left(x_{n}-p\right)+\beta_{n}\left(T^{n} x_{n}-p\right), \tau_{n}\right\rangle \\
& \leq\left\|\left(1-\beta_{n}\right)\left(x_{n}-p\right)+\beta_{n}\left(T^{n} x_{n}-p\right)\right\|^{2}+\left\|\tau_{n}\right\|^{2} \\
& =\left(1-\beta_{n}\right)\left\|x_{n}-p\right\|^{2}+\beta_{n}\left\|T^{n} x_{n}-p\right\|^{2}-\beta_{n}\left(1-\beta_{n}\right)\left\|T^{n} x_{n}-x_{n}\right\|^{2}+\left\|\tau_{n}\right\|^{2} .
\end{aligned}
$$

hence

$$
\begin{aligned}
\left\|y_{n}-p\right\|^{2} \leq & 2\left(1-\beta_{n}\right)\left\|x_{n}-p\right\|^{2}+2 \beta_{n}\left\|T^{n} x_{n}-p\right\|^{2}+2\left\|\tau_{n}\right\|^{2}-2 \beta_{n}\left(1-\beta_{n}\right)\left\|T^{n} x_{n}-x_{n}\right\|^{2} \\
\leq & 2\left(1-\beta_{n}\right)\left\|x_{n}-p\right\|^{2}+2 \beta_{n}\left[\left(1+\gamma_{n}\right)\left\|x_{n}-p\right\|^{2}+k_{n}\left\|T^{n} x_{n}-x_{n}\right\|^{2}+c_{n}\right] \\
& +2\left\|\tau_{n}\right\|^{2}-2 \beta_{n}\left(1-\beta_{n}\right)\left\|T^{n} x_{n}-x_{n}\right\|^{2} \\
= & 2\left(1+\beta_{n} \gamma_{n}\right)\left\|x_{n}-p\right\|^{2}+2 \beta_{n} c_{n}+2\left\|\tau_{n}\right\|^{2}-2 \beta_{n}\left(1-\beta_{n}-k_{n}\right)\left\|T^{n} x_{n}-x_{n}\right\|^{2} \\
\leq & 2\left(1+\beta_{n} \gamma_{n}\right)\left\|x_{n}-p\right\|^{2}+2 \beta_{n} c_{n}+2\left\|\tau_{n}\right\|^{2}-2 \delta^{2}\left\|T^{n} x_{n}-x_{n}\right\|^{2} .
\end{aligned}
$$

Which implies that

$$
\left\|y_{n}-p\right\|^{2} \leq 2\left(1+\beta_{n} \gamma_{n}\right)\left\|x_{n}-p\right\|^{2}+2 \beta_{n} c_{n}+2\left\|\tau_{n}\right\|^{2} .
$$

By condition (i), (ii) and (7), we have $\left\{\left\|y_{n}-p\right\|\right\}$ be bounded, and so $\left\{y_{n}\right\}$.

From lemma 4, we obtained

$$
\left\|T^{n} y_{n}-p\right\|^{2} \leq \frac{1}{1-k_{n}}\left(k_{n}\left\|y_{n}-p\right\|+\sqrt{1+\left(1-k_{n}\right) \gamma_{n}\left\|y_{n}-p\right\|^{2}+\left(1-y_{n}\right) c_{n}}\right),
$$

hence $\left\{\left\|T^{n} y_{n}-p\right\|\right\}$ be bounded. By $\left\|y_{n}-T^{n} y_{n}\right\| \leq\left\|T^{n} y_{n}-p\right\|+\left\|y_{n}-p\right\|$, we have $\left\{\left\|y_{n}-T^{n} y_{n}\right\|\right\}$ be bounded.

Next we prove that $\lim _{n \rightarrow \infty}\left\|x_{n}-p\right\|$, for each $p \in F(T)$ exists. Indeed,

$$
\begin{aligned}
\left\|x_{n+1}-p\right\|^{2}= & \left\|\left(1-\alpha_{n}\right)\left(x_{n}+\sigma_{n}-p\right)+\alpha_{n}\left(T^{n} y_{n}+\sigma_{n}-p\right)\right\|^{2} \\
\leq & \left(1-\alpha_{n}\right)\left\|x_{n}+\sigma_{n}-p\right\|^{2}+\alpha_{n}\left\|T^{n} y_{n}+\sigma_{n}-p\right\|^{2}-\alpha_{n}\left(1-\alpha_{n}\right)\left\|T^{n} y_{n}-x_{n}\right\|^{2} \\
\leq & \left(1-\alpha_{n}\right)\left\|x_{n}-p\right\|^{2}+2\left(1-\alpha_{n}\right)\left\langle\sigma_{n}, x_{n}+\sigma_{n}-p\right\rangle+\alpha_{n}\left\|T^{n} y_{n}-p\right\|^{2} \\
& +2 \alpha_{n}\left\langle\sigma_{n}, T^{n} y_{n}+\sigma_{n}-p\right\rangle-\alpha_{n}\left(1-\alpha_{n}\right)\left\|T^{n} y_{n}-x_{n}\right\|^{2} \\
\leq & \left(1-\alpha_{n}\right)\left\|x_{n}-p\right\|^{2}+2\left(1-\alpha_{n}\right)\left\langle x_{n}-p, \sigma_{n}\right\rangle+\alpha_{n}\left\|T^{n} y_{n}-p\right\|^{2} \\
& +2 \alpha_{n}\left\langle T^{n} y_{n}-p, \sigma_{n}\right\rangle+2\left\langle\sigma_{n}, \sigma_{n}\right\rangle-\alpha_{n}\left(1-\alpha_{n}\right)\left\|T^{n} y_{n}-x_{n}\right\|^{2} \\
= & \left(1-\alpha_{n}\right)\left\|x_{n}-p\right\|^{2}+\alpha_{n}\left\|T^{n} y_{n}-p\right\|^{2}+2\left\langle\left(1-\alpha_{n}\right)\left(x_{n}-p\right)+\alpha_{n}\left(T^{n} y_{n}-p\right), \sigma_{n}\right\rangle \\
& +2\left\|\sigma_{n}\right\|^{2}-\alpha_{n}\left(1-\alpha_{n}\right)\left\|T^{n} y_{n}-x_{n}\right\|^{2} .
\end{aligned}
$$

In the other hand,

$$
\begin{aligned}
2\left\langle\left(1-\alpha_{n}\right)\left(x_{n}-p\right)+\alpha_{n}\left(T^{n} y_{n}-p\right), \sigma_{n}\right\rangle \leq & \left\|\left(1-\alpha_{n}\right)\left(x_{n}-p\right)+\alpha_{n}\left(T^{n} y_{n}-p\right)\right\|^{2}+\|\sigma\|^{2} \\
\leq & \left(1-\alpha_{n}\right)\left\|x_{n}-p\right\|^{2}+\alpha_{n}\left\|T^{n} y_{n}-x_{n}\right\|^{2} \\
& -\alpha_{n}\left(1-\alpha_{n}\right)\left\|T^{n} y_{n}-x_{n}\right\|^{2}+\left\|\sigma_{n}\right\|^{2}
\end{aligned}
$$

By substituting (10) into (9), we have

$$
\begin{aligned}
\left\|x_{n+1}-p\right\|^{2} \leq & 2\left(1-\alpha_{n}\right)\left\|x_{n}-p\right\|^{2}+2 \alpha_{n}\left\|T^{n} y_{n}-p\right\|^{2}-2 \alpha_{n}\left(1-\alpha_{n}\right)\left\|T^{n} y_{n}-x_{n}\right\|^{2}+3\left\|\sigma_{n}\right\|^{2} \\
\leq & 2\left(1-\alpha_{n}\right)\left\|x_{n}-p\right\|^{2}+2 \alpha_{n}\left[\left(1+\gamma_{n}\right)\left\|y_{n}-p\right\|^{2}+k_{n}\left\|y_{n}-T^{n} y_{n}\right\|^{2}+c_{n}\right] \\
& -2 \alpha_{n}\left(1-\alpha_{n}\right)\left\|T^{n} y_{n}-x_{n}\right\|^{2}+3\left\|\sigma_{n}\right\|^{2} .
\end{aligned}
$$

Substituting (6) into (11), we have,

$$
\begin{aligned}
\left\|x_{n+1}-p\right\|^{2} \leq & 2\left[\left(1-\alpha_{n}\right)+\alpha_{n}\left(1+\gamma_{n}\right)\left(1+\beta_{n} \gamma_{n}\right)\right]\left\|x_{n}-p\right\|^{2}+2 \alpha_{n}\left(1+\gamma_{n}\right)\left(\beta_{n} c_{n}+2\left\|\tau_{n}\right\|^{2}\right) \\
& +2 \alpha_{n} c_{n}+3\left\|\sigma_{n}\right\|^{2}+2 \alpha_{n} k_{n}\left\|y_{n}-T^{n} y_{n}\right\|-2 \delta^{2}\left\|T^{n} y_{n}-x_{n}\right\|^{2}-2 \delta^{2}\left\|x_{n}-T^{n} x_{n}\right\|^{2} \\
\leq & 2 \varepsilon_{n}\left\|x_{n}-p\right\|^{2}+\theta_{n}-2 \delta^{2}\left\|T^{n} y_{n}-x_{n}\right\|^{2}-2 \delta^{2}\left\|x_{n}-T^{n} x_{n}\right\|^{2} .
\end{aligned}
$$

where $\varepsilon_{n}=\left[\left(1-\alpha_{n}\right)+\alpha_{n}\left(1+\gamma_{n}\right)\left(1+\beta_{n} \gamma_{n}\right)\right], \theta_{n}=2 \alpha_{n}\left(1+\gamma_{n}\right)\left(2 \beta_{n} c_{n}+3\left\|\tau_{n}\right\|^{2}\right)+2 \alpha_{n} c_{n}+3\left\|\sigma_{n}\right\|^{2}+2 \alpha_{n} k_{n}\left\|y_{n}-T^{n} y_{n}\right\|$. Hence,

$$
\left\|x_{n+1}-p\right\|^{2} \leq 2 \varepsilon_{n}\left\|x_{n}-p\right\|^{2}+\theta_{n}
$$


By the conditions (i), $\Sigma_{n=1}^{\infty}\left(\varepsilon_{n}-1\right)<\infty, \Sigma_{n=1}^{\infty} \theta_{n}<\infty$, and Lemma 1, we have

$$
\lim _{n \rightarrow \infty}\left\|x_{n}-p\right\| \text { exists. }
$$

On the other hand, from (12) we have,

$$
\delta^{2}\left\|T^{n} y_{n}-x_{n}\right\|^{2} \leq \varepsilon_{n}\left\|x_{n}-p\right\|^{2}-\left\|x_{n+1}-p\right\|^{2}+\theta_{n} .
$$

By the conditions (i) and the boundeness of $\left\{x_{n}\right\}$, we have

$$
\lim _{n \rightarrow \infty}\left\|T^{n} y_{n}-x_{n}\right\|=0 .
$$

Again by (12),

$$
\delta^{2}\left\|T^{n} x_{n}-x_{n}\right\|^{2} \leq \varepsilon_{n}\left\|x_{n}-p\right\|^{2}-\left\|x_{n+1}-p\right\|^{2}+\theta_{n} .
$$

Hence,

$$
\lim _{n \rightarrow \infty}\left\|T^{n} x_{n}-x_{n}\right\|=0
$$

and

$$
\left\|x_{n+1}-x_{n}\right\|=\left\|\alpha_{n}\left(T^{n} y_{n}-x_{n}\right)+\sigma_{n}\right\| \leq \alpha_{n}\left\|T^{n} y_{n}-x_{n}\right\|+\left\|\sigma_{n}\right\| .
$$

From (15) and condition (i),

$$
\lim _{n \rightarrow \infty}\left\|x_{n+1}-x_{n}\right\|=0
$$

By (16), (17) and uniformly continous of $T^{n}$, by Theorem 2 we obtain that

$$
\lim _{n \rightarrow \infty}\left\|x_{n}-T x_{n}\right\|=0 .
$$

By the boundedness of $\left\{x_{n}\right\}$, there exists a subsequence $\left\{x_{n_{j}}\right\} \subseteq\left\{x_{n}\right\}$ such that $\left\{x_{n_{j}}\right\}$ converges weakly to $x$. From (16), uniform continuits of $T$ and theorem 2, we see that

$$
\lim _{n \rightarrow \infty}\left\|x_{n}-T^{m} x_{n}\right\|=0
$$

for all $m \in \mathbb{N}$. We obtain $x \in F(T)$.

Next we prove the $\omega_{\omega}\left(\left\{x_{n}\right\}\right)=\{x\}$. Suppose there exists another subsequence $\left\{x_{n_{k}}\right\} \subseteq\left\{x_{n}\right\}$ which converges to $z \neq x$. As in the case of $x$, we must have $z \in F(T)$ It follows from (11) that $\lim _{n \rightarrow \infty}\left\|x_{n}-x\right\|$ and $\lim _{n \rightarrow \infty}\left\|x_{n}-z\right\|$ exist. Since $\left\{x_{n}\right\}$ satisfies the Opial condition, we have

$$
\begin{aligned}
\lim _{n \rightarrow \infty}\left\|x_{n}-x\right\| & =\lim _{k \rightarrow \infty}\left\|x_{n_{k}}-x\right\|<\lim _{k \rightarrow \infty}\left\|x_{n_{k}}-z\right\|=\lim _{n \rightarrow \infty}\left\|x_{n}-z\right\| \\
& =\lim _{j \rightarrow \infty}\left\|x_{n_{j}}-z\right\|<\lim _{j \rightarrow \infty}\left\|x_{n_{j}}-x\right\|=\lim _{n \rightarrow \infty}\left\|x_{n}-x\right\|,
\end{aligned}
$$

a contradiction. So that $z=x$ and $\omega_{\omega}\left(\left\{x_{n}\right\}\right)=\{x\}$. Thus, $\left\{x_{n}\right\}$ converges weakly to $x$.

Remark 1. Theorem 2 and Theorem 3 are more general than the results studied in D.R.Sahu [2009] and Schu [1991].

\section{References}

B.E.Rhoades. (1994). Fixed point iterations for certain nonlinear mappings, J.Math.Anal.Appl., 183, 118-120.

C.E.Chidume, E.U.Ofoedu, H.Zegeye. (2003). Strong and weak convergence theorems for asymptotically nonexpansive mappings, J.Math.Anal.Appl., 280, 364-374.

D.R.Sahu, H.K.Xu, J.C.Yao. (2009). Asymptotically strict pseudocontractive mappings in the intermediate sense, Nonlinear.Anal., 70, 3502-3511.

J.Schu. (1991). Weak and strong convergence of fixed points of asymptotically nonexpansive mapping, J.Math.Anal.Appl., $159,407-413$. 
J.Schu. (1991). Weak and strong convergence of fixed points of asymptotically nonexpansive maps, Bull.Austral.Math.Soc., 43, 153-159.

K.Goebel, W.A.Kirk. (1972). A fixed point theorem for asymptoticall nonexpansive mappings, Pro.Amer.Math.Soc., 35(1), 171-174.

K.K.Tan, H.K.Xu. (1992). Nonlinear ergodic theorems for asymptotically nonexpansive mappings in Banach spaces, Proc.Amer.Math.Soc., 114, 399-404.

K.Nammanee, M.A.Noor, S.Suantai. (2000). Convergence criteria of modified Noor iterations with errors for asymptotically nonexpansive mappings, J.Math.Model, 32, 1182-1191.

L.Wang. (2006). Strong and weak convergence theorems for common fixed points of noneself asymptotically nonexpansive mappings, J.Math.Anal.Appl., 323(1), 550-557.

M.O.Osilike, S.C.Aniagbosor. (2000). Weak and strong convergence theorems for fixed points of asymptotically nonexpansive mappings, Math.Comput.Model, 32, 1182-1192.

R.E.Bruck, T.Kuczumow, S.Reich. (1993). Convergence of iterates of asymptotically nonexpansive mappings in Banach spaces with the uniform Opial property, Colloq.Math, 65, 169-179.

R.P.Agarwal, Doneal O'Regan, D.R.Sahu. (2007). Iterative construction of fixed points of nearly asymptotically nonexpansive mappings, J.Nonlinear Convex Anal., 8(1), (61-67).

S.H.Khan, H.Fukharuddin. (2005). Weak and strong convergence of a scheme with errors for two nonexpansive mappings, Nonlinear Anal., 61, 1295-1301.

S.S.Chang, Y.J.Cho, H.Zhou. (2001). Demi-closed principle and weak convergence problems for asymptotically nonexpansive mappings, J.Korean Math,Soc., 38, 1245-1260.

T.H.Kim, H.K.Xu. (2008). Convergence of the modified Mann's iteration method for asymptotically strict pseudocontractions, Nonliner Anal., 68, 2828-2836.

Z.Q.Liu, S.M.Kang. (2004). Weak and strong convergence for fixed points of asymptotically nonexpansive mappings, Acta Math Sinica, 20, 1009-1018. 